\title{
Le rayonnement synchrotron, une source de lumière dédiée à la recherche
}

Marie-Emmanuelle Couprie ${ }^{(i)}$, Jean-Claude Denard (i) (jean-claude.denard@synchrotron-soleil.fr), Laurent Farvacque $^{(2)}$, Gaël Le Bec ${ }^{(2)}$, Amor Nadji( ${ }^{(1)}$ et Jean-Luc Revol ${ }^{(2)}$ (revolj|@esrf.fr)

(1) Synchrotron SOLEll, Saint-Aubin, BP 48, 91192 Gif-sur-Yvette Cedex

(2) ESRF, BP 220, 38043 Grenoble Cedex

Les électrons relativistes qui circulent dans les accélérateurs de particules produisent un rayonnement intense.

Ce rayonnement synchrotron couvre un domaine en énergie allant des ondes millimétriques aux rayons $X$ durs avec une brillance exceptionnelle, des polarisations linéaires ou circulaires, et une structure temporelle exploitable.

\section{En France, deux centres}

de ce type sont en

fonctionnement [1] :

I'European Synchrotron

Radiation Facility (ESRF),

situé à Grenoble, et SOLEIL (a), implanté sur le plateau de Saclay, à Saint-Aubin (Essonne).

(a) SOLEIL : acronyme de "Source Optimisée de Lumière d'Énergie Intermédiaire du LURE ". LURE : "Laboratoire d'Utilisation du Rayonnement Synchrotron ». Ce laboratoire d'Orsay a disparu en 2003, pour être remplacé par la société civile "Synchrotron SOLEIL ». Principe de production
et caractéristiques du rayonnement synchrotron

Quand un électron est accéléré, il rayonne de l'énergie électromagnétique [2]. Si des électrons se déplacent à faible vitesse sur une trajectoire circulaire, ils émettent un rayonnement monochromatique dans toutes les directions. Dans les accélérateurs de particules, les électrons ont une vitesse très proche de celle de la lumière. D'après la relativité restreinte, leur masse augmente fortement et, pour un observateur immobile, les longueurs apparaissent plus courtes que dans le référentiel en mouvement. La longueur d'onde $\lambda$ du rayonnement est très réduite : l'ESRF produit des rayons $\mathrm{X}$ durs, dont l'énergie va de quelques $\mathrm{keV}$ à plus de $100 \mathrm{keV}(\boldsymbol{\lambda}=0,013 \mathrm{~nm})$. Les photons sont émis dans la direction du mouvement des particules, dans un cône dont l'ouverture est d'autant plus petite que l'énergie des électrons est grande. Ce rayonnement est appelé rayonnement synchrotron.

Les premiers utilisateurs de rayonnement synchrotron se sont installés sur des collisionneurs, parallèlement aux expériences de physique des particules [3]. Ils ont tiré parti de ce rayonnement parasite dès les années 60 , sur ces sources dites de première génération.

La deuxième génération fut entièrement dédiée aux utilisateurs de rayonnement synchrotron. Ces sources furent construites dans les années 70 et 80 . Comme dans les sources de première génération, le rayonnement synchrotron était produit dans les aimants de courbure qui dévient les électrons pour les maintenir sur une trajectoire fermée. Un électron qui passe dans un tel aimant crée une impulsion électromagnétique de durée très courte (fig. 2a). Un flux de photons plus élevé est obtenu en insérant des aimants de polarités alternées pour cumuler l'effet des déviations. Ce type d'assemblage est appelé un wiggler.

Les onduleurs (fig. 1a) sont des sources de rayonnement plus intenses. Ce sont des assemblages périodiques d'aimants, comme les wigglers. Mais, dans les onduleurs [3], la déflexion des électrons est plus faible (fig. 2b) et les photons émis à chaque période interferent : le spectre du rayonnement est discret, et composé d'une fréquence fondamentale et d'harmoniques. Un onduleur $[4,5]$ rayonne dans un cône plus fermé que celui d'un aimant de courbure ou d'un wiggler. Les sources de troisième génération, telles que l'ESRF et SOLEIL, ont été optimisées pour permettre l'installation de nombreux onduleurs. Toutefois, les photons émis par différents électrons ne sont pas cohérents temporellement, car la longueur des paquets d'électrons est largement supérieure aux longueurs d'onde du rayonnement.

La longueur d'onde des photons émis par les onduleurs est accordable, en faisant varier l'amplitude du champ magnétique. Si le champ magnétique périodique augmente, la déviation des électrons est plus importante et ils mettent plus de temps à parcourir une période : la longueur 


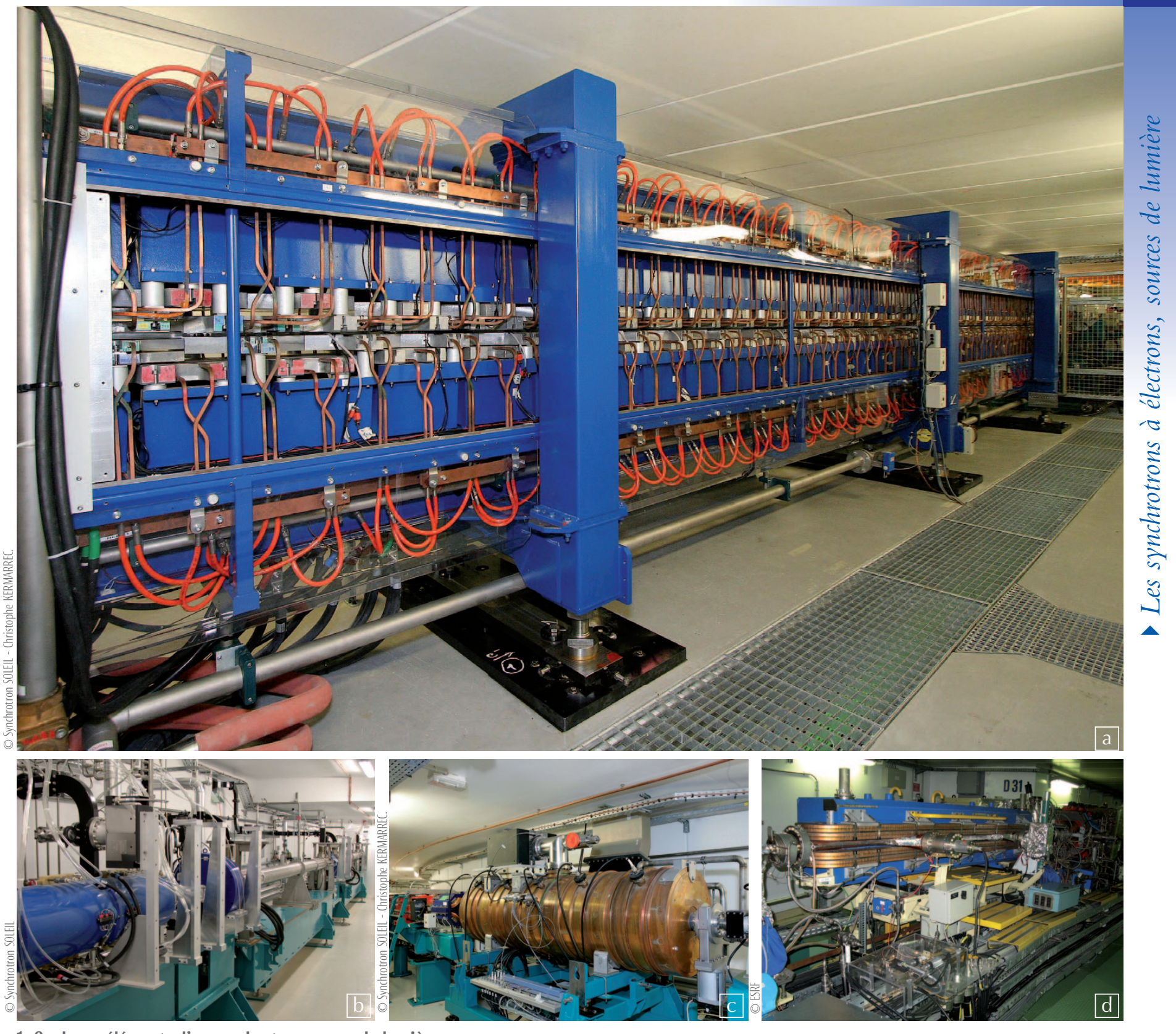

1. Quelques éléments d'un synchrotron source de lumière.

(a) Un onduleur : HU 640 de la ligne DESIRS de SOLEIL, dans la gamme d'énergie 5 à 50 eV (ultraviolet). (b) L'accélérateur linéaire (LINAC) de SOLEIL. (c) La cavité radiofréquence du booster de SOLEIL. (d) Un aimant de courbure de l’anneau de stockage de l'ESRF.

d'onde du rayonnement augmente. Les assemblages magnétiques des onduleurs sont optimisés pour satisfaire les besoins des utilisateurs : certains onduleurs ont une faible plage de réglage tandis que d'autres, moins performants à une énergie donnée, sont accordables dans une large gamme d'énergie. La polarisation des photons est déterminée par la trajectoire des électrons. Suivant la configuration du champ magnétique, cette trajectoire est sinusoïdale ou hélicoïdale. La polarisation est alors linéaire, elliptique ou circulaire. Les onduleurs sont réalisés à partir d'aimants permanents très puissants. $\mathrm{La}$ brillance spectrale, définie comme le nombre de photons émis par unité de
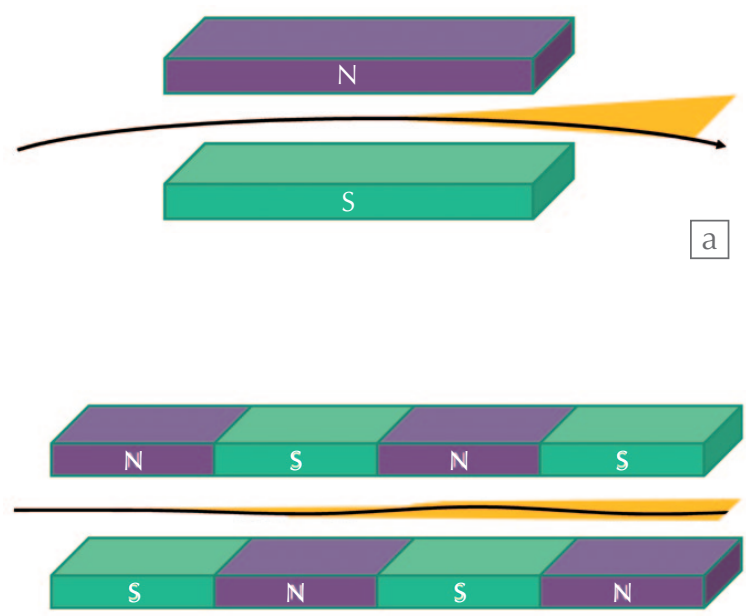

$\mathrm{b}$
2. Rayonnement émis par une source synchrotron.

(a) Rayonnement d'un aimant de courbure (dipôle) : deux pôles, nord et sud, créent un champ magnétique homogène. Un électron qui passe dans ce champ magnétique est soumis à une accélération transverse et est dévié. Des photons sont émis tangentiellement à la trajectoire, dans un cône de faible ouverture (en jaune).

(b) Rayonnement d'un onduleur l'électron est dévié par une série d'aimants. Le cône dans lequel est émis le rayonnement est beaucoup plus étroit pour un onduleur que pour un dipôle. 


\section{>>}

temps, de bande passante, de taille et de divergence, est une caractéristique importante des sources de rayonnement synchrotron (fig. 3).

Les électrons sont regroupés en paquets, dont le nombre maximum est fonction de la circonférence de l'anneau. Le nombre de paquets dépend de la structure temporelle souhaitée : l'anneau peut être rempli de manière uniforme ou être partiellement rempli. Le rayonnement reçu par une ligne de lumière n'est donc pas continu, mais consiste en une suite d'impulsions espacées de quelques nanosecondes à quelques microsecondes. La durée des impulsions est de l'ordre de quelques dizaines de picosecondes pour les anneaux de stockage de troisième génération. La taille et la divergence $\mathrm{du}$ faisceau de photons rayonnés sont directement liées à la taille et à la divergence du faisceau d'électrons. Le produit de ces deux grandeurs est appelé l'émittance. L'émittance est constante le long de l'anneau ; c'est une caractéristique importante des sources de rayonnement synchrotron.

Pour les expériences à très haute résolution spatiale ou de diffusion cohérente, il est nécessaire d'avoir une émittance aussi faible que possible. Des sources de troisième génération ont dès à présent une émittance verticale très faible et fournissent des faisceaux proches de la limite de diffraction dans cette direction, jusque dans les rayons $\mathrm{X}$ durs. L'avènement d'anneaux à très faible émittance horizontale va conduire à de nouvelles sources de troisième génération qui, d'ici une dizaine d'années, atteindront

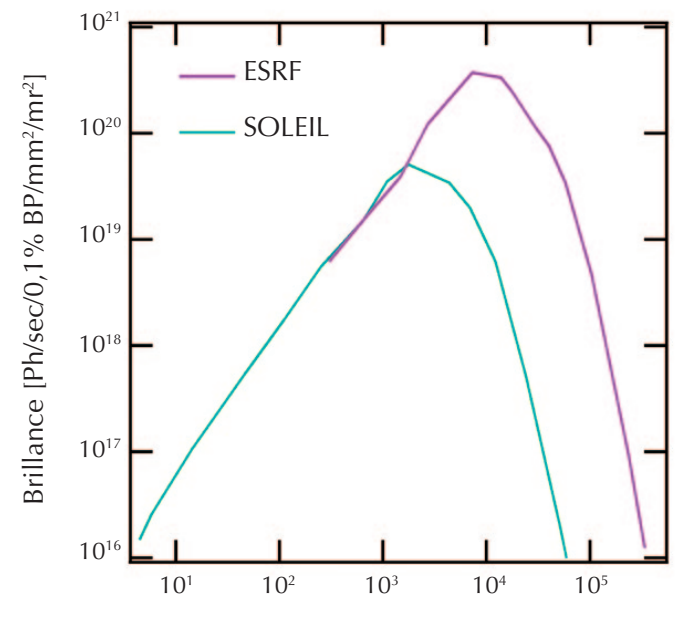

3. Brillances spectrales de l'ESRF et de SOLEIL.

La brillance, définie comme le nombre de photons émis par unité de temps, de bande passante, de taille et de divergence, est une caractéristique importante des sources de rayonnement synchrotron. Cette figure a été obtenue en superposant les courbes de brillance d'onduleurs et de wigglers représentatifs des deux machines. $\odot$ J. Chavanne.

Énergie des photons $[\mathrm{eV}]$ des besoins expérimentaux. Sur un périmètre de $844 \mathrm{~m}$, l'ESRF dispose de 28 sections droites dédiées, équipées de près de 90 onduleurs de différents types. Les utilisateurs sont répartis sur 42 lignes de lumière, dont 12 utilisent le rayonnement produit par les aimants de courbure. L'ESRF fonctionne de façon continue 24 heures sur 24, six jours par semaine pour les utilisateurs et un jour par semaine pour la maintenance et le développement. En 2011, les 42 lignes ont utilisé le faisceau durant plus de 5000 heures, avec une disponibilité proche de $99 \%$ et un temps moyen de 108 heures entre deux pannes. Depuis sa création, l'ESRF a bénéficié d'une politique d'amélioration des accélérateurs et des onduleurs. Depuis 2009, l'institut implémente un important programme de jouvence concernant les lignes de lumière, les infrastructures et les accélérateurs [6].

À SOLEIL [1], l'exploitation des premières lignes de lumière a démarré en 2006. En 2013, les utilisateurs disposeront de plus de 5000 heures de faisceau sur 24 lignes de lumière, dans un mode de fonctionnement similaire à celui de l'ESRF. Les travaux se poursuivent pour achever la mise au point et la construction d'autres lignes, pour arriver à un total de 29. La conception compacte de l'anneau de stockage permet de réserver plus de $42 \%$ de sa circonférence (354 m) aux onduleurs. Les performances de SOLEIL sont optimisées dans une gamme d'énergie complémentaire à celle de l'ESRF.

\section{Principales caractéristiques des accélérateurs de l'ESRF et de SOLEIL}

L'ESRF et SOLEIL sont deux installations complémentaires, dont les principales caractéristiques sont données dans le tableau p. 21. L'énergie plus importante des électrons de l'ESRF fournit davantage de flux de photons $\mathrm{X}$ de hautes énergies, entre 4 et $100 \mathrm{keV}$ (fig. 3). SOLEIL accueille des expériences couvrant une très grande plage du spectre, depuis les ondes millimétriques jusqu'aux rayons $\mathrm{X}$ $(\lambda \leq 0,03 \mathrm{~nm})$, en passant par l'infrarouge, le visible et l'ultraviolet. Exprimée en énergie des photons par la relation $E=h v=h c / \lambda$, où h est la constante de Planck et $\mathrm{c}$ la vitesse de la lumière, cette gamme s'étend de $1,5 \mathrm{meV}$ à $50 \mathrm{keV}$. 


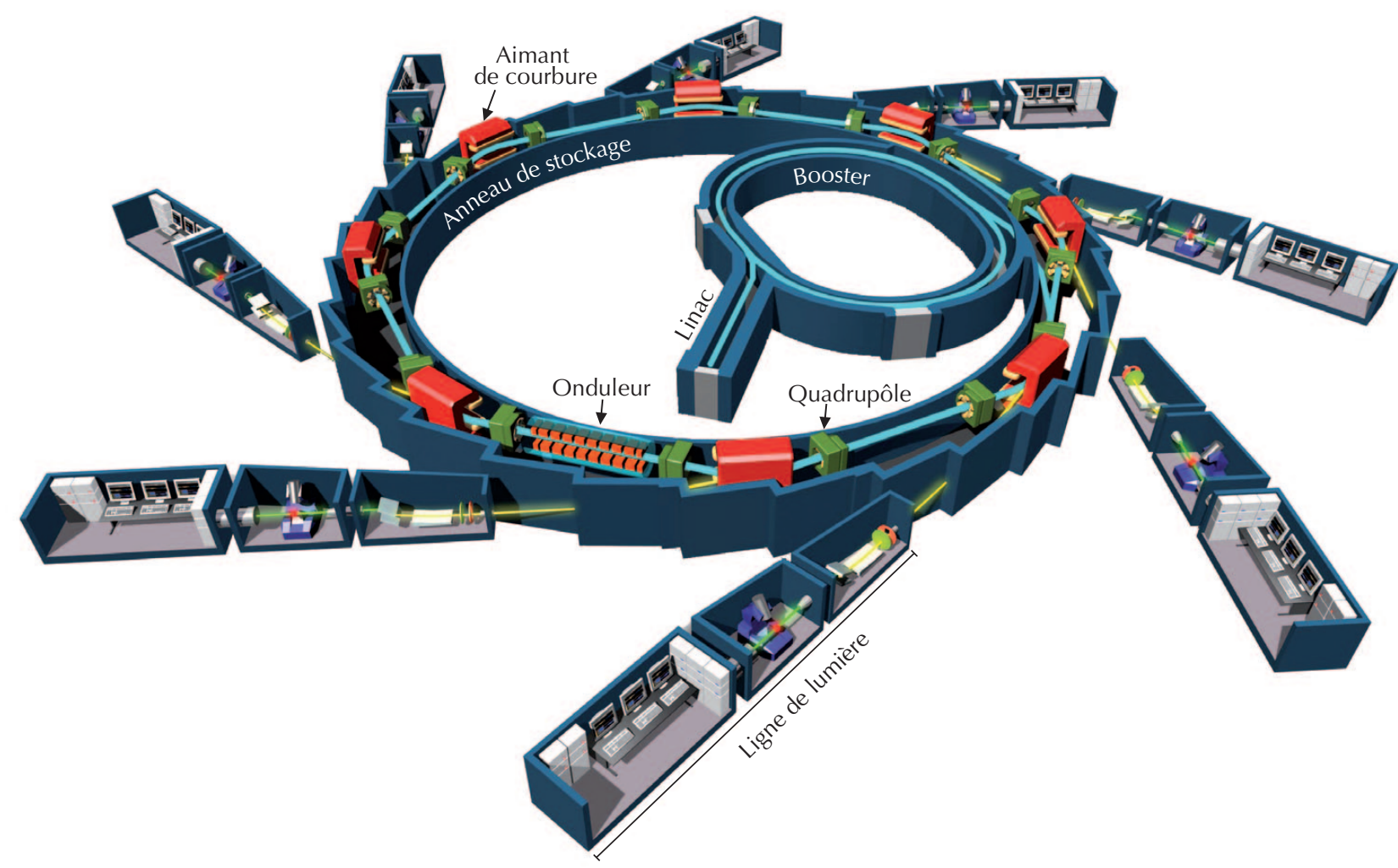

4. Schéma de principe d’une installation synchrotron. Les électrons émis par le canon sont accélérés dans le LINAC, puis dans le booster pour être ensuite injectés dans l'anneau de stockage. Après accumulation, ils produisent du rayonnement synchrotron dans les aimants de courbure (dipôles, en rouge) et les onduleurs. Ce rayonnement est utilisé par un grand nombre de lignes de lumière, construites tangentiellement à l’orbite des électrons, pour des expériences scientifiques très variées.

La configuration des deux installations est semblable (fig. 4). Une cathode thermo-ionique émet des électrons qui sont accélérés dans un accélérateur linéaire (le LINAC, fig. 1b) de quelques dizaines de mètres. À ce point, les électrons atteignent une énergie de $100 \mathrm{MeV}$ à SOLEIL et $200 \mathrm{MeV}$ à l'ESRF. Les électrons sont ensuite injectés dans un accélérateur circulaire, appelé "booster". Le booster est équipé de cavités radiofréquence (352 MHz) qui produisent un champ électrique permettant aux électrons de gagner de l'énergie à chaque tour (fig. 1c). Seuls les électrons qui arrivent en phase avec l'oscillation du champ radiofréquence sont maintenus en orbite stable. Ceci provoque le regroupement des particules en paquets équidistants. Les électrons sont accélérés jusqu'à leur énergie nominale et peuvent alors être injectés dans l'anneau de stockage (beaucoup plus grand que le booster), où ils sont maintenus à énergie constante. Ils circulent sur la même orbite pendant plusieurs heures dans un tube où règne un vide très poussé, de l'ordre de $10^{-9}$ mbar. Le processus d'injection se répète plusieurs fois par seconde, ce qui permet d'accumuler dans l'anneau de stockage un courant atteignant une fraction d'ampère $(200 \mathrm{~mA}$ à l'ESRF et $430 \mathrm{~mA}$ à SOLEIL).

Pour fournir de l'énergie aux électrons, il faut appliquer un champ électrique dans leur direction de propagation ;

Principaux paramètres de l'ESRF et de SOLEIL dans les conditions d'exploitation de 2012.

\begin{tabular}{|c|c|c|}
\hline Paramètres & ESRF & SOLEIL \\
\hline Énergie des électrons (GeV) & 6,04 & 2,75 \\
\hline Circonférence de l'anneau (m) & 844 & 354 \\
\hline Courant dans l'anneau (mA) & 200 & 430 \\
\hline Émittance horizontale (nm.rad) & 4 & 3,9 \\
\hline Émittance verticale (nm.rad) & 0,005 & 0,04 \\
\hline Longueur des paquets (ps) & 20 & 20 \\
\hline Disponibilité (2011) & $98,6 \%$ & $98,4 \%$ \\
\hline
\end{tabular}

pour dévier leur trajectoire, on utilise des champs magnétiques constants dans l'anneau de stockage. En fait, l'anneau de stockage n'est pas circulaire, mais a la forme d'un polygone, constitué de segments rectilignes ("sections droites"), sur lesquels sont installés les "éléments d'insertion ", onduleurs et wigglers (fig. 5). À la jonction de deux sections droites se trouvent les aimants de courbure (fig. 1d), aussi appelés dipôles, qui apportent les $360^{\circ}$ nécessaires à la trajectoire pour en faire une orbite fermée. La structure magnétique de l'anneau comprend aussi des quadrupôles (fig. 6a), qui ramènent les électrons sur l'orbite moyenne, et des hexapôles (fig. 6b), qui corrigent des aberrations. Les caractéristiques de ces aimants définissent l'optique magnétique de la machine, dont un des paramètres principaux est l'émittance horizontale du faisceau d'électrons. L'émittance dans le plan vertical est beaucoup plus faible et peut être corrigée, car elle provient uniquement des imperfections de champs magnétiques.

Les électrons ne circulent pas indéfiniment dans l'anneau de stockage. Quelques-uns se perdent lors des chocs avec des molécules de gaz résiduel présentes dans le tube à vide, ou lors de chocs 
>>

entre eux à l'intérieur des paquets d'électrons. Ces pertes sont moins importantes à l'ESRF qu'à SOLEIL, car les électrons ont une énergie plus élevée, ce qui rend le faisceau plus "rigide». SOLEIL a mis en œuvre un système d'injections fréquentes, dit top-up, qui consiste à rajouter une bouffée d'électrons dès que le courant a baissé d'une quantité prédéterminée ( $0,5 \%$ à SOLEIL, soit une injection toutes les trois minutes environ). Ainsi, le courant est quasi constant et les faisceaux de photons délivrés aux expériences sont plus stables, car la charge thermique sur les miroirs des lignes de lumière est constante. On appelle durée de vie le temps qu'il faudrait pour perdre tous les électrons s'ils continuaient à se perdre au même rythme. Avec une durée de vie de l'ordre de 45 heures, l'ESRF n'a pas besoin de fonctionner en top-up. Une injection toutes les douze heures est suffisante pour limiter la variation du courant à moins de $20 \%$ de sa valeur nominale.

Dans sa conception, SOLEIL a bénéficié des améliorations technologiques des anneaux de stockage antérieurs et en a apporté de nouvelles à la communauté des sources de lumière. L'ultravide, qui intervient sur la durée de vie du faisceau, est amélioré par un alliage de titane-zirconium-vanadium déposé sur la surface interne de la chambre à vide sur la majeure partie de la circonférence de l'anneau. À SOLEIL, des puissances accélératrices de l'ordre du mégawatt sont fournies par des transistors plutôt que par des tubes à vide. Une nouvelle électronique très performante permet de mesurer précisément l'orbite des électrons en 122 points, de façon à la stabiliser à court et à long terme à mieux que le micromètre, par l'intermédiaire d'asservissements d'orbite. L'électronique, développée en collaboration avec l'industrie, est maintenant utilisée dans d'autres centres (dont l'ESRF). La stabilité du faisceau est une caractéristique très importante pour les utilisateurs, et l'asservissement de l'orbite n'en est que l'ultime étape. À SOLEIL, par exemple, la dalle de béton supportant l'anneau mesure $80 \mathrm{~cm}$ d'épaisseur et repose, 15 mètres plus bas, soutenue par 600 pieux de béton, sur la couche de sable fin et compact. D'importantes précautions ont été prises dans la conception et l'utilisation des équipements de l'infrastructure (ponts roulants, compresseurs, moteurs, fluides de refroidissement, liquéfacteur d'hélium, etc.) Enfin, le mode d'injection top-up, mentionné précédemment contribue aussi à la stabilité.

\section{Perspectives des installations de rayonnement synchrotron en France}

\section{Les anneaux de stockage à très petite émittance}

Les évolutions récentes des connaissances de dynamique du faisceau d'électrons et de la technologie des accélérateurs donnent naissance à des projets d'anneaux ayant une émittance horizontale très faible (10 pm.rad) [6], qui approche celle de la limite de diffraction des photons dans le domaine des rayons $\mathrm{X}$ durs, jusqu'à $10 \mathrm{keV}$. La plus grande partie du faisceau sera cohérente transversalement. L'ESRF propose un projet intermédiaire [8], qui utilise le tunnel existant tout en conservant la position des lignes de lumière. L'émittance horizontale serait réduite de 4 à 0,16 nm.rad, associée à une augmentation de la brillance et de la cohérence d'un facteur 30 pour des énergies de l'ordre de $50 \mathrm{keV}$. Si le projet est accepté, sa réalisation est envisagée pour 2018.

\section{Machines compactes \\ de type ThomX}

Les récents progrès dans les technologies des lasers et des accélérateurs permettent d'envisager l'utilisation de l'effet de rétrodiffusion Compton [9] comme source ultra-compacte de rayons $\mathrm{X}$, par collision frontale d'un faisceau laser intense (visible ou IR) avec un faisceau d'électrons relativistes. De telles sources peuvent produire un flux relativement important de rayons X pulsés. La relation univoque entre l'énergie des photons rétrodiffusés et l'angle de collision permet d'obtenir un flux quasi monochromatique par un simple système de diaphragmes.

Le projet ThomX [10], en construction au Laboratoire de l'Accélérateur Linéaire d'Orsay, produira un flux de $10^{12}$ à $10^{13} \mathrm{ph} / \mathrm{s}$, avec une divergence angulaire de quelques mrad et une dispersion en énergie d'environ $10 \%$ pour des rayons X d'énergie maximale de quelques dizaines de $\mathrm{keV}$. Si le flux intégré de photons n'est pas aussi élevé que celui des synchrotrons, le coût et les dimensions de ce type de machines les rendent attractives pour de nombreuses applications. L'encombrement de la machine ThomX est d'environ $70 \mathrm{~m}^{2}$; elle pourrait être installée dans un hôpital pour traiter des patients sur place, ou dans un musée pour analyser des pièces qui ne peuvent être transportées.

5. Organisation typique d'une section droite de l'anneau de stockage. Dans une source de rayonnement synchrotron, l'optique magnétique de l'anneau de stockage est conçue pour laisser périodiquement des espaces nécessaires à l'installation des onduleurs. Sur la figure, la section droite est occupée par trois onduleurs (en violet). Le premier à gauche est un onduleur sous vide (voir la photo de la page 6). 


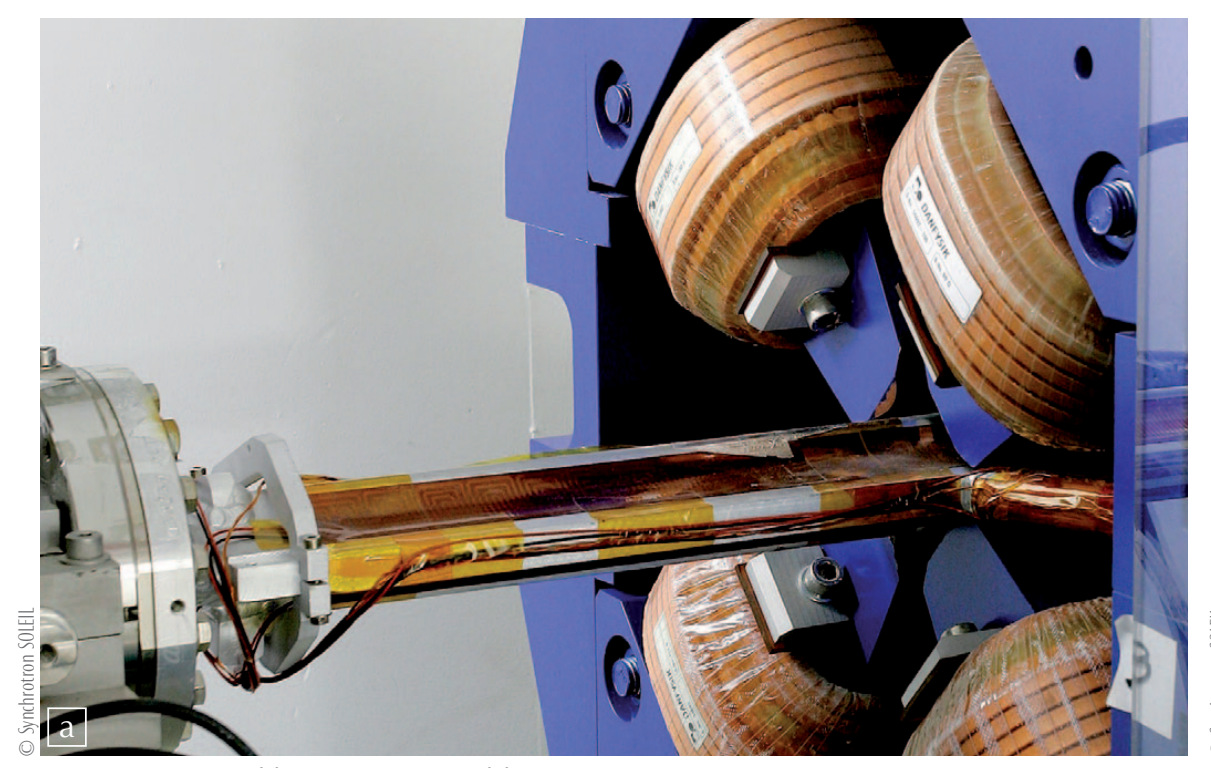

6. Un quadrupôle (a) et un sextupôle (b) de l'anneau de stockage de SOLEIL.

\section{Sources laser à électrons libres (LEL)}

Les sources de quatrième génération sont complémentaires des anneaux de rayonnement synchrotron. Elles ciblent les études de la dynamique des processus ou l'accès aux informations pour les espèces très diluées. Plusieurs lasers à électrons libres [11], dont deux fonctionnent dans le domaine des rayons $\mathrm{X}$, sont opérationnels dans le monde.

Il y a un LEL à Orsay, CLIO (Centre serveur Laser à électrons libres dans l'Infrarouge d'Orsay) ; cependant, il n'y a en France aucune installation de type LEL de courte longueur d'onde, ni en fonctionnement ni en construction.

Le projet LUNEX5 [12] (Laser à électrons libres Utilisant un accélérateur Nouveau pour Exploitation de rayonnement $\mathrm{X}$ de $5^{\mathrm{e}}$ génération) vise à produire des impulsions courtes, intenses et cohérentes dans le domaine des rayons $\mathrm{X}$ mous (40 à $4 \mathrm{~nm}$ ). Ce démonstrateur comporte deux types de machines : un accélérateur linéaire supraconducteur permettant d'évoluer en haute cadence vers des branches LEL multiples, et un accélérateur laser plasma à faible taux de répétition, qui sera qualifié par l'application laser à électrons libres. Les deux faisceaux d'électrons alimentent une ligne LEL commune comportant des onduleurs sous vide. Deux expériences pilotes résolues en temps pourront profiter du rayonnement de LUNEX5 et démontrer ses performances.

\section{Conclusion}

Les sources de lumière synchrotron basées sur les anneaux de stockage ont atteint avec la troisième génération une très grande maturité. L'exploitation des dernières avancées technologiques et l'amélioration constante du fonctionnement ont permis à des sources comme l'ESRF et SOLEIL de produire une lumière d'une extrême brillance, dix mille milliards de fois plus forte que l'anode tournante des tubes à rayons X, avec une stabilité et une disponibilité des plus remarquables. La faible émittance du faisceau et la diversité des onduleurs ont ouvert de nouvelles perspectives offrant aux utilisateurs de toutes disciplines des moyens de caractérisation, d'analyse et d'imagerie exceptionnels.

En proposant de diminuer encore l'émittance horizontale du faisceau d'un ordre de grandeur, ces sources vont continuer à jouer un rôle clé en offrant des propriétés complémentaires aux lasers à électrons libres. Ces derniers, fournissant maintenant les premiers faisceaux lasers X femtoseconde accordables, ouvrent des perspectives nouvelles pour les expériences résolues en temps avec des impulsions ultracourtes.

\section{Remerciements}

Merci à Joël Chavanne pour les courbes de brillances des deux anneaux de stockage, et à Marie-Pauline Gacoin pour son aide en suggérant les corrections appropriées après relecture.

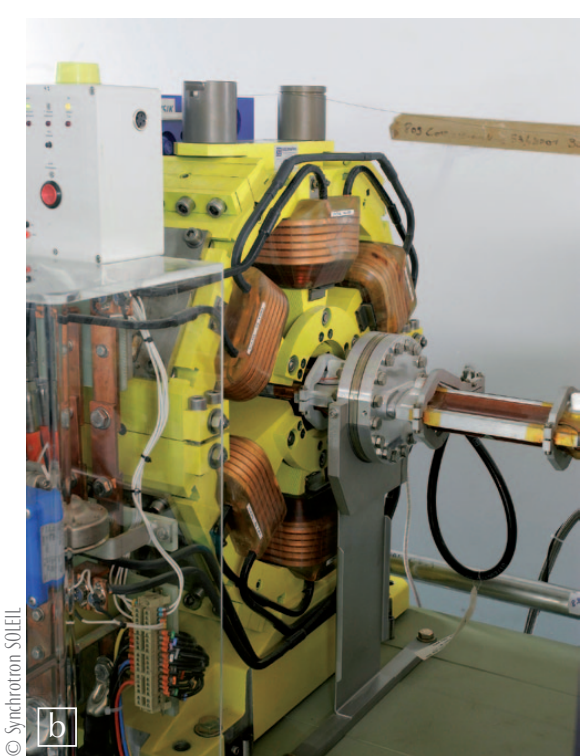

\section{Références}

1• Site web de I'ESRF : www.esrf.eu/ Site web de SOLEIL : www.synchrotron-soleil.fr/

2• R. Feynman, R. Leighton et M. Sands, The Feynman Lectures in Physics, Vol. 1, ch. 34, Addislon-Wesley Publishing Company (1864).

3- Pour approfondir la physique des accélérateurs de particules, le rayonnement synchrotron et le fonctionnement des lasers à électrons libres H. Wiedermann, Particle Accelerator Physics (3e édition), Springer (2007).

4 R.P. Walker, "Insertion devices: undulators and wigglers", Synchrotron radiation and free electron lasers, CERN 98-04 (1998), pp. 129-190

5• H. Onuki et P. Elleaume, Undulators, wigglers and their applications, Taylor \& Francis (2003)

6• H. Reichert, dans ce numéro, p. 14.

7- Référence pour avoir plus de détails sur la machine de SOLEIL : J.M. Filhol et al., "Commissioning of the Storage Ring of SOLEIL, the French $3^{\text {rd }}$ Generation Synchrotron Radiation Facility", IFCA Beam Dynamics Newsletter 44 (2007) 160.

8• J.L. Revol et al., "Performance and upgrade of the ESRF light source" www.ipac2011.org/pre_press/THPC009.PDF

9• V. Malka et al., Reflets de la physique 33 (2013) 23-26.

10• "ThomX Conceptual Design Report", http://hal.archives-ouvertes.fr/docs/00/44/82/ 78/PDF/ThomXCDRAV.pdf

11- FLASH (DESY, Hambourg) : W. Ackermann et al., Nature Photonics 1 (2007) 336 ; ScSS (Japon) T. Shintake et al., ibid. 2 (2008) 555 ; LCLS (Stanford, USA) : P. Emma et al., ibid. 4 (2010) 641 ; SACLA (Japon) : T. Ishikawa et al., ibid. 6 (2012) 540 ; FERMI (Trieste, Italie) : E. Allaria et al., ibid. 6 (2012) 699.

12 - M.E. Couprie et al., "The LUNEX5 project in France", J. Phys.: Conf. Ser. 425 (2013) 072001 